\title{
Amblyomma dissimile (Acari: Ixodidae) PARÁSITO DE BOa constrictor EN COLOMBIA
}

\section{Amblyomma dissimile (Acari: I xodidae) PARASI TE OF Boa constrictor I N COLOMBIA}

\author{
Juan Carrascal V, ${ }^{1 *}$ M.Sc, Teresa Oviedo S, ${ }^{1}$ M.Sc, Santiago Monsalve B, ${ }^{1}$ Esp, \\ Ana Torres $M,{ }^{2}$ Bióloga.
}

\begin{abstract}
${ }^{1}$ Universidad de Córdoba, Facultad de Medicina Veterinaria y Zootecnia. Departamento de Ciencias Pecuarias. Montería, Colombia. ${ }^{2}$ Universidad San Martín, Facultad de Medicina Veterinaria y Zootecnia. Fundación Neotrópica Colombia. Armenia, Colombia. *Correspondencia: jcvelasque@yahoo.com
\end{abstract}

Recibido: Marzo 24 de 2009; Aceptado: Junio 25 de 2009.

\section{RESUMEN}

Objetivo. Describir algunas garrapatas encontradas en dos ejemplares de Boa constrictor, llevados al Centro de Atención y Valoración de Fauna Silvestre en Montería, Córdoba, Colombia. Materiales y métodos. Se recolectaron 37 garrapatas provenientes de dos individuos adultos de Boa constrictor, los parásitos fueron conservados en alcohol al $70 \%$ y posteriormente identificados mediante diversas claves taxonómicas. Resultados. Todas las garrapatas fueron identificadas como Amblyomma dissimile, de las cuales, 9 fueron hembras, 24 machos y 4 ninfas. Conclusiones. La identificación de ectoparásitos en especies de Boa c. constrictor contribuye a mantener adecuadamente esta especie en cautiverio y provee datos para establecer medidas profilácticas y tratamiento, igualmente, ayuda en el conocimiento de los agentes parasitarios de la fauna silvestre.

Palabras clave: Amblyomma dissimile, Boa constrictor, Colombia.

\section{ABSTRACT}

Objective. To describe ticks found in two Boa constrictor specimens. Materials and methods. 37 ticks were collected from two adults of Boa constrictor, the parasites were preserved in alcohol $70 \%$ and later identified through various keys. The specimens were brought to the Center for Care and Assessment of Wildlife in Montería, Córdoba, Colombia. Results. All the ticks were identified as Amblyomma dissimile, of which 9 were females, 24 males and 4 nymphs. Conclusions. The identification of species of ectoparasites of Boa constrictor contribute to properly maintain this specie in captivity and provides data to establish preventive measures and treatment, also helps in understanding the parasitic agents of wildlife.

Key words: Amblyomma dissimile, Boa constrictor, Colombia. 


\section{NTRODUCCIÓN}

Las garrapatas (Arácnida: Ixodoidea) son ácaros de gran tamaño (2-30 mm) ectoparásitos hematófagos obligados, en los estados postembrionales, de una amplia gama de vertebrados terrestres y voladores.

En el mundo se han descrito cerca de 850 especies, divididas en tres familias, Argasidae, Nuttalliellidae e Ixodidae. La familia I xodidae se divide en Prostriata y Metastriata. Prostriata comprende alrededor de 240 especies correspondientes al género Ixodes; y Metastriata se divide en cuatro subfamilias: Amblyomminae, Haemaphysalinae, Hyalomminae y Rhipicephalinae (1). La subfamilia Amblyomminae agrupa los géneros Amblyomma y Aponomma. De las aproximadamente 106 especies del género Amblyomma, 57 se distribuyen en la región neotropical y 37 de éstas parasitan reptiles (2).

Ixodidae ejercen en el hospedero una acción mecánica, causante de daños ulcerativos en la dermis, mucosa y órganos anexos, que pueden ser colonizados por hongos, bacterias o larvas de dípteros (3) o posibilitar la entrada de endoparásitos (4). También ejercen acción expoliatriz capaz de ocasionar anemia severa y pueden funcionar como vectores de protozoos hemoparásitos, como hemogregarinas (5), filarias, y retrovirus causantes de la enfermedad por cuerpos de inclusión (6).

Los ectoparásitos más frecuentes de los boidos son las garrapatas y los ácaros, aunque de estos últimos sólo ha sido reportada una especie, Ophionyssus natricis. Estos artrópodos se localizan preferentemente en el área alrededor de la cloaca y en la cabeza, especialmente en el pliegue de piel delgada, muy vascularizada, que aparece entre el anteojo y las escamas perioculares y también en los cantos externos de los párpados, donde pueden provocar irritación corneal, queratitis e incluso disecdisis (7). Como la presencia de estos ectoparásitos resulta muy molesta, el hospedador puede permanecer mucho tiempo dentro del agua o rascándose contra bordes, lo que puede ocasionar heridas en la boca, nariz y ojos.

El objetivo del presente trabajo fue determinar especimenes de garrapatas encontradas en dos ejemplares de Boa constrictor.

\section{MATERI ALES Y MÉTODOS}

Las garrapatas obtenidas en el estudio provenían de dos ejemplares de Boa constrictor llevados al Centro de Atención y Valoración de Fauna Silvestre - (CAV) de la Corporación del Valle del rio Sinú y San Jorge (CVS) de Montería - Colombia. Estas fueron retiradas manualmente y conservadas en alcohol al $70 \%$ para su posterior identificación.

Las garrapatas adultas fueron identificadas utilizando las siguientes claves: Clave para identificación de garrapatas y Clave para identificación de géneros de garrapatas de la familia I xodidae y Argasidae (8); Clave para familias de garrapatas (9); Clave para la identificación de las especies de Amblyomma del hemisferio occidental (10) y clave para las especies venezolanas del género Amblyomma (11).

Las ninfas fueron clasificadas tentativamente por asociación con los caracteres morfológicos de los adultos encontrados en el mismo hospedero.

\section{RESULTADOS}

De los dos ejemplares de Boa constrictor se retiraron un total de 37 garrapatas. Todos los individuos fueron identificados como Amblyomma dissimile Koch, 1844, de los cuales 9 fueron hembras, 24 machos y 4 ninfas (Tabla 1). 
Tabla 1. Número de garrapatas en dos ejemplares de B. constrictor

\begin{tabular}{ccccc}
\hline Hospedero & \multicolumn{4}{c}{ Número } \\
\cline { 2 - 5 } (Boa constrictor) & Hembras & Machos & Ninfas & Total \\
\hline 1 & 3 & 2 & $4^{*}$ & 9 \\
2 & $6^{*}$ & 22 & & 28 \\
\hline
\end{tabular}

* Una ninfa recuperada del hospedero 1 y una hembra procedente del hospedero 2 no presentaron gnatosoma.

\section{SCUSIÓN}

En el neotrópico se han encontrado 6 especies de Amblyomma parásitas de B. constrictor, A. fuscum Neumann, 1907, que parece estar relacionado exclusivamente con esta serpiente (12), A. argentinae Neumann, 1904 (13), A. quadricavum, Schulze, 1941, A. scutatum Neumann, 1899 (14), A. dissimile y A. rotundatum Koch, 1844, (15)

A. dissimile ha sido frecuentemente reportada en Boa constrictor $(16,17)$ incluso bajo condiciones de laboratorio (18). Según Adis (19) esta especie es un ectoparásito de común presentación en anfibios y reptiles, con una amplia distribución geográfica, que va desde los Estados Unidos de América hasta el sur de la Argentina. Algunos lugares de colección son México, Paraguay, Puerto Rico, Guyana Francesa, El Salvador, Antigua y Barbuda, Barbados, Belice, Brasil, Colombia, Costa Rica, Grenada, Guatemala, Guyana, Honduras, Jamaica, Nicaragua, Panamá, Perú, Santa Lucía, Trinidad y Tobago, Venezuela, Guadalupe, Cuba, República Dominicana, Haití y Ecuador. Aunque también se han encontrado ejemplares en el Neártico (2).

Aragão y Fonseca (20) discutieron la validez de algunas especies de Amblyomma e insistieron en que un $A$. diminutivum colectados en serpientes de Brasil y Colombia era sinónimo de $A$. dissimile.

A. dissimile tiene como hospederos naturales reptiles y anfibios, como Bufo marinus, Boa imperatus, Iguana tuberculata, Epicrates cenchria (21), Callopsis areolata, C. pulcherrima y C. rubida (3), Ameiva ameiva, Boa constrictor, Chironius carinatus, Crotalus durissus, Dryadophis sp., I guana iguana y Pseudoboa neuwiedii (11). No obstante, también se ha encontrado en mamíferos, como bovinos y roedores de las familias Hidrochaeridae y Echimyidae (22). Experimentalmente se ha logrado infestar conejos, Oryctolagus cuniculus, con ninfas de esta especie (22).

A pesar de que $A$. dissimile tiene menor importancia económica comparada con otras especies de garrapatas, se han reportado lesiones severas en hospederos gravemente infestados (22). Por otra parte, es importante recordar la importancia que estos parásitos pueden tener en la transmisión de enfermedades, ya que a nivel experimental se ha logrado utilizar larvas de A. dissimile como vectores de Cowdria ruminantium (22) e individuos adultos como vectores de Hepatozoon fusifex (23).

La identificación de los principales ecto y endoparásitos de Boa c. constrictor, es de gran valor para una adecuada manutención en cautiverio, por proveer información para el diseño de medidas profilácticas y el tratamiento de estos agentes patológicos. Pero también para hacer inferencias respecto de la fauna parasitaria de las poblaciones silvestres, el posible papel de las garrapatas en la transmisión de endoparásitos, y considerar este aspecto en el diseño de planes de conservación.

Es de gran interés retomar el estudio de especies de garrapatas de menor importancia económica y sanitaria, su distribución, hospederos, ciclos de vida entre otros aspectos, de fauna silvestre, porque los registros en Colombia son antiguos, y en su mayoría corresponden a hallazgos fortuitos. 
Además los cambios ambientales impuestos por la agricultura y la ganadería favorecen ciertas especies e indudablemente alteran o incluso llevan a la extinción asociaciones ancestrales garrapata-hospedero, y en el caso especial de $A$. dissimile porque los sitios de colecta son distantes y sus hospederos múltiples.

\section{Agradecimientos}

Al personal que labora en el Centro de Atención y Valoración de Fauna Silvestre CAV de la Corporación Autónoma de los Valles del Sinú y San J orge - CVS. Montería, Colombia.

\section{REFERENCI AS}

1. Black WC, Piesman J. Phylogeny of hard- and soft-tick taxa (Acari: Ixodida) based on mitochondrial 16S rDNA sequences. Proc Natl Acad Sci USA 1994; 91: 10034-8.

2. Guglielmone AA, Estrada-Peña A, Keirans JE, Robbins RG. Ticks (Acari: I xodida) of the Neotropical Zoogeographic Region. International Consortium on Ticks and Tick-borne Diseases (ICTTD-2). Atalanta, Houten: The Netherlands; 2003.

3. Ernst $\mathrm{CH}$, Ernst EM. Ectoparasites associated with Neotropical Turtles of the Genus Callopsis (Testudines, Emydidae, Batagurinae). Biotropica 1977; 9(2): 139-142.

4. Orós J. Enfermedades víricas en serpientes. En: Memorias VII Congreso Anual de la Sociedad Española de Medicina Interna Veterinaria. Las Palmas de Gran Canaria; 2001.

5. Molina-Prescott I, López R, Molleda J M, Martín E, Quevedo MA, Díaz-Paniagua $C$, et al. Hematología y Bioquímica Sanguínea en el Camaleón común (Chamaeleo chamaeleon). En: Memorias VII Congreso Anual de la Sociedad Española de Medicina Interna Veterinaria. Las Palmas de Gran Canaria. 2001.

6. Barragán K. Enfermedades de Reptiles y Anfibios. Boletín GEAS. 2002. 3(1-6): 18-27.
7. Bayón del Río A. Afecciones Oculares en Reptiles. Memorias VII Congreso Anual de la Sociedad Española de Medicina Interna Veterinaria. Las Palmas de Gran Canaria. 2001.

8. Bequaert JC. The ticks, or Ixodoidea of northeastern United States and Eastern Canada. Entomol Am New Ser 1946; 25: 73-232.

9. Strickland RK, Gerrish RR, Hourrigan J L, Schubert GO. Ticks of veterinary importance. Agriculture Handbook $\mathrm{N}^{\circ}$ 485. Washington D.C: United States Departament of Agriculture; 1976.

10. López VG, Parra G. Amblyomma neumanni, Ribaga 1902. Primera comprobación en Colombia y Claves para las especies de Amblyomma. Revista ICA 1985; 20: 152-162.

11. Guerrero R. Las garrapatas de Venezuela (Acarina: Ixodoidea). Listado de especies y claves para su identificación. Bol Dir Malariol San Amb 1996; 36(1 y 2): 1-24.

12. Durden LA, Knapp CR. Ticks parasitizing reptiles in the Bahamas. Med Vet Entomol 2005; 19(3): 326 - 328.

13. Guglielmone AA, Luciani C, Mangold AJ . Aspects of the ecology of Amblyomma argentinae Neumann, 1904 [ = Amblyomma testudinis (Conil, 1877)] (Acari: Ixodidae). Systematic \& Applied Acarology Special Publications. 2001; 8: 1-12 
14. Burridge MJ, Simmons LA. Exotic ticks introduced into the United States on imported reptiles from 1962 to 2001 and their potential roles in international dissemination of diseases. Vet Parasitol 2003; 113(3-4): 289-320.

15. Boero JJ. Los I xodoideos de la republica de argentina y sus huespedes. Rev fac agron Vet 1954; 13: 505-515

16. Vogelsang EG, Cordero EH. Sobre una pequeña colección de garrapatas (Ixodidae) de la Argentina, Paraguay y Uruguay. Rev Med Vet Parasitol 1939; 1:115-116.

17. Ivancovich JC, Luciani CA. Las garrapatas de Argentina. Monogr Asoc Arg Parasitol Vet 1992, 95.

18. Dunn LH. Studies on the iguana tick Amblyomma dissimile, in Panama. J Parasitol 1918; 5: 1-10.

19. Adis J. Oservacões eco-entomológicas da Amazonia: I Um carrapato ectoparasito da Boa constrictor. Acta Amazonica 1981; 11:407.
20. Aragão HB, Fonseca F. Notas de Ixodologia. V. A proposito da validade de algumas espécies do gênero Amblyomma do continente americano (Acari-Ixodidae). Mem Inst Oswaldo Cruz 1953; 51: 485-492.

21. Osorno ME. Las Garrapatas de la República de Colombia. Rev Facultad Nacional de Agronomía 1942; 16 .

22. Freitas LHT, Faccini JLH, Daemon $E$, Prata MCA, Barros-Battesti DM. Experimental infestation with the immatures of Amblyomma dissimile Koch, 1844 (Acari:lxodidae) on Tropidurus torquatus (Lacertilia: I guanidae) and Oryctolagus cuniculus. Arq Bras Med Vet Zootec 2004; 56(1): 126-129.

23. Ball GH, Chao J, Telford SR Jr. Hepatozoon fusifex sp.n., a hemogregarine from Boa constrictor producing marked morphological changes in infected erythrocytes. J Parasitol 1969; 55: 800-813. 